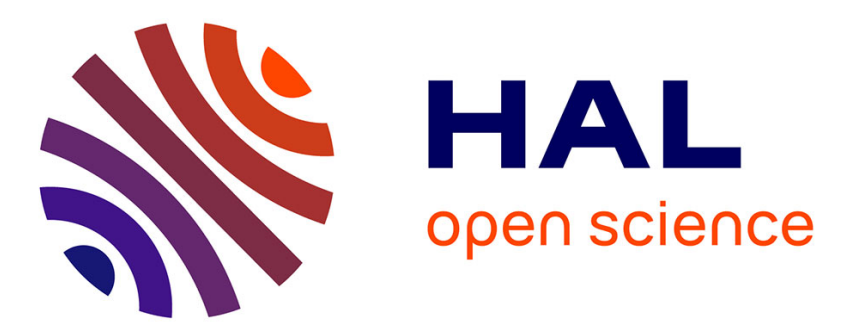

\title{
EFFECT OF ORDER-PARAMETER COLLECTIVE MODES ON NMR AND ULTRASOUND ATTENUATION IN 3He-A
}

\author{
L. Tewordt, N. Schopohl
}

\section{- To cite this version:}

L. Tewordt, N. Schopohl. EFFECT OF ORDER-PARAMETER COLLECTIVE MODES ON NMR AND ULTRASOUND ATTENUATION IN 3He-A. Journal de Physique Colloques, 1978, 39 (C6), pp.C6-6-C6-8. 10.1051/jphyscol:1978603 . jpa-00217495

\section{HAL Id: jpa-00217495 https://hal.science/jpa-00217495}

Submitted on 1 Jan 1978

HAL is a multi-disciplinary open access archive for the deposit and dissemination of scientific research documents, whether they are published or not. The documents may come from teaching and research institutions in France or abroad, or from public or private research centers.
L'archive ouverte pluridisciplinaire HAL, est destinée au dépôt et à la diffusion de documents scientifiques de niveau recherche, publiés ou non, émanant des établissements d'enseignement et de recherche français ou étrangers, des laboratoires publics ou privés. 


\title{
EFFECT OF ORDER-PARAMETER COLLECTIVE MODES ON NMR AND ULTRASOUND ATTENUATION IN ${ }^{3}$ He-A
}

\author{
L. Tewordt and N. Schopoh1
}

Abteilung für Theoretische Festkörperphysik, Universität Homburg, D-2000 Hamburg 13, Germany

Résumé.- Les largeurs de bande du mode clapping (spin) dues à la rupture des paires se trouvent être p1us étroites que celles du mode flapping (orbital). Un champ magnétique provoque un éclatement des fréquences de tous les modes. Les modes orbitaux ont un effet appréciable sur les largeurs de bande et les glissements de bande du NMR lorsque $T$ est très bas et $T$ très près de $T$. L'attênuation des ultrasons est appréciablement augmentée par les modes flapping même pour $\vec{q} \| \vec{l}$.

Abstract.- The line widths of the c1apping (spin) modes due to pair-breaking are found to be much smaller than those of the flapping (orbital) modes. A magnetic field causes a splitting of the frequencies of all these modes. The orbital modes have an appreciable effect on the line widths and line shifts of the NMR at very low $T$ and at $T$ very $c$ close to $T_{c}$. The ultrasound attenuation is enhanced appreciably by the flapping modes even for $\vec{q} \| \vec{l}$.

1. INTRODUCTION.- We have extended a previous calculation /1/ of the spin and density correlation functions for ${ }^{3} \mathrm{He}-\mathrm{A}$ at temperature $\mathrm{T}=0$ and magnetic field $H=O$ to all $\mathrm{T}$ and to finite $\mathrm{H}$. The coupling of the 9 components $d_{\alpha i}$ of the order parameter by means of the magnetic dipole interaction is taken into account rigorously. The results are valid for a11 frequencies $\omega$, including $\omega \sim \Delta_{0}(\mathrm{~T})$ where $\Delta_{\mathrm{o}}(\mathrm{T})$ is the maximum of the energy gap. The direction of the wave vector $\vec{q}$ is restricted to lie along the equilibrium anisotropy axis $\vec{l}$. While quasi-particle collisions are neglected, damping by pair-breaking processes is taken into account. Our results reduce to those derived by previous theories in their 1imited range of validity, i.e. either $\omega \ll \Delta_{0}(T)$ (references $/ 2,3 /$ ) or neglect of dipole interaction and $q \neq 0$. (reference $/ 4 /$ ).

\section{COLLECTIVE MODES.- The longitudina1 and trans-} verse dynamic spin susceptibilities yield, beside the ordinary longitudinal NMR at $\omega_{D}$ and the transverse NMR at $\omega_{t}=\left(\omega_{D}^{2}+\omega_{L}^{2}\right)^{1 / 2}$, resonances at frequencies of the order of the gap frequency, $\Delta_{0}(T)$. For the "flapping" modes the vector $\vec{l}$ oscillates about the fixed direction of the vector $\vec{d}$ either in the plane of $\vec{d}$ and $\vec{H}$ or perpendicular to this plane. Here $\vec{l}$ describes the orientation of the orbital angular momentum of the Cooper pairs and $\vec{d}$ describes the orientation of the spin axes. For $q=0$ we find at low and high temperatures for the frequency, $\omega_{n f l}$ ' and the line width, $\Gamma_{\mathrm{nf} 1}$, of the "normal-flapping" mode (in the following $\Delta_{0} \equiv \Delta_{0}(T)$ ) :

$\omega_{\mathrm{nf1}}^{2} \stackrel{n_{1}}{=} \omega_{\ell}^{2}+\frac{(4 / 9)}{\ln \left(4 \Delta_{0} / \omega_{\ell}\right)}(\pi \mathrm{T})^{2} ;$

$$
\begin{aligned}
& \Gamma_{\mathrm{nfl}} \cong(\pi / 10) \frac{\omega_{\mathrm{nf} 1}}{\ln \left(4 \Delta_{0} / \omega_{\ell}\right)} ;\left(\mathrm{T} \ll \mathrm{T}_{\mathrm{c}}\right) \\
& \left(\omega_{\mathrm{nf1}} / 2 \Delta_{0}\right)^{2}=\frac{3}{5}-\frac{2}{5}\left[1-5\left(\omega_{\ell} / 2 \Delta_{0}\right)^{2}\right]^{1 / 2} ; \\
& \Gamma_{\mathrm{nf1}}=(\sqrt{5} / \pi) \omega_{\mathrm{nf} 1} ;\left(\mathrm{T} \lesssim \mathrm{T}_{\mathrm{c}}\right) .
\end{aligned}
$$

The dipole-flapping frequency $\omega_{\ell}$ is of order $\omega_{D}$ :

$\omega_{\ell}^{2} \cong \frac{(3 / 2) \omega_{D}^{2}}{\left(1+F_{0}^{a}\right) \ln \left(4 \Delta_{0} / \omega_{D}\right)} ;(T=0)$.

$\omega_{l}^{2}=\frac{32}{\pi^{2}} \frac{\omega_{D}^{2}}{\left(1+F_{0}^{a}\right)} \frac{T_{c}}{\Delta_{0}} ;\left(T \lesssim T_{c}\right)$.

For the so called "super-flapping" modes we obtain frequencies $\omega_{s f l}$ and line widths $\Gamma_{\text {sfl }}$ which are given by :

$\omega_{\text {sf1 }}^{2}=\left(1.58 \Delta_{0}\right)^{2} ; \Gamma_{s f 1} \cong \pi \omega_{s f 1} ;(T=0)$.

$\left(\omega_{s f 1} / 2 \Delta_{0}\right)^{2}=\frac{3}{5}+\frac{2}{5}\left[1-5\left(\omega_{\ell} / 2 \Delta_{0}\right)^{2}\right]^{1 / 2} ;$

$\Gamma_{\text {sf1 }}=(3 / \pi) \omega_{\text {sf1 }} ;\left(T \lesssim T_{c}\right)$.

One sees that for $\left(1-T / T_{c}\right) \lesssim 10^{-8}$ there exist no flapping modes.

The third category of high-frequency modes are the so called "clapping" modes where the vector $\vec{d}$ oscillates about the fixed direction of the vector $\vec{l}$ either in the plane of $\vec{\ell}$ and $\overrightarrow{\mathrm{H}}$ or perpendicular to this plane. The frequency, $\omega_{\mathrm{cl}}$, and the line width, $\Gamma_{c 1}$, of the former mode which is due to the transverse susceptibility are equal to

$\omega_{\mathrm{cl}}^{2}=\left(1.22 \Delta_{0}\right)^{2}+\omega_{\mathrm{L}}^{2} ; \Gamma_{\mathrm{cl}} \cong(\pi / 13) \omega_{\mathrm{c} 1} ;(\mathrm{T}=0)$. $\omega_{\mathrm{c} 1}^{2}=(1 / 3) \omega_{\ell}^{2}+\left(1.12 \Delta_{0}\right)^{2}+\omega_{\mathrm{L}}^{2} ; \Gamma_{\mathrm{c} 1}=0.06 \omega_{\mathrm{c} 1} ;$ 
$\left(\mathrm{T} \leqslant \mathrm{T}_{\mathrm{c}}\right)$.

For the clapping mode due to the longitudinal susceptibility where $\vec{d}$ oscillates in the plane perpendicular to $\vec{H}$ the same expression holds apart from the fact that the Larmor frequency term $\omega_{L}^{2}$ is absent.

It is interesting to notice that the line widths of the normal- and super-flapping modes are so large that these modes are not well-defined. The line widths of the clapping modes, however, are about an order of magnitude smaller. The reason is that the clapping modes the effect of the pair-breaking processes in the vicinity of the nodes of the gap is strongly suppressed.

3. EFFECT ON NMR. - The oscillator strengths of the high frequency modes arising from the dynamic spin susceptibilities are smaller by a factor of the order of the dipole interaction strength,

$\mu \equiv\left(36 \pi \gamma^{2} / 5 \mathrm{~N}(0) \mathrm{g}^{2}\right) \sim 10^{-6}$ than the oscillator strengths of the ordinary NMR at $\omega_{D}$ and $\omega_{t}$. Resonance effects are to be expected whenever $\omega_{t}$ matches one of the (temperature dependent) collective mode frequencies, that is, $\omega_{n f l}$ or $\omega_{s f l}$ (the condition $\omega_{t}=\omega_{c 1}$ can never be satisfied as is clear from the expression of the transverse $\omega_{c 1}$ is given above).

The effect of the normal flapping mode on the NMR has been considered previously by Leggett and Takagi /2/ and by Combescot/3/. Due to the large damping term, $\Gamma_{n f l}$, we do not find two NMR peaks near resonance of the flapping mode and the transverse NMR $\left(\left|w_{t}^{2}-w_{n f 1}^{2}\right| \leqslant \omega_{D}^{2}\right)$, in contrast to reference $/ 2 \%$. But we find that the transverse NMR width, $\Gamma_{t}$, considered as a function of temperature is peaked at a high temperature, say $T_{1}$, and at a low temperature, say $T_{2}$, where $T_{1}$ and $T_{2}$ are given by the resonance condition $\omega_{n f 1}=\omega_{t}$. Our results for the NMR line widths and line shifts caused by the normal flapping mode at low temperatures agree essentially with those obtained in reference /3/ for this temperature range. However, as has been shown in reference $/ 3 /$, these effects become observable only at extremely low temperatures. While the kinetic theories of references $/ 2 /$ and $/ 3 /$ are restricted to $\omega \ll \Delta_{0}(\mathrm{~T})$, our theory is valid for all $\omega$. Therefore it is applicable also near $T_{c}$ where $\omega_{n f 1} \sim \Delta_{0}(T)$. For instance, for the shift of the longitudinal NMR frequency, $\Delta \omega_{D}$, we obtain near $T_{c}$ : $\left(\Delta \omega_{D} / \omega_{D}\right)=-\left(2 / \pi^{2}\right)\left(1+F_{o}^{a}\right)^{-1}\left(T_{c} / \Delta_{0}\right)\left(\omega_{D} / \omega_{n f 1}\right)^{2}$. This line shift is opposite in direction to that caused by spin relaxation effects $/ 3 /$. For $\left(1-\mathrm{T} / \mathrm{T}_{\mathrm{c}}\right)<10^{-2}$ the former effect becomes dominant over the latter one. Further, the width of the transverse NMR is :

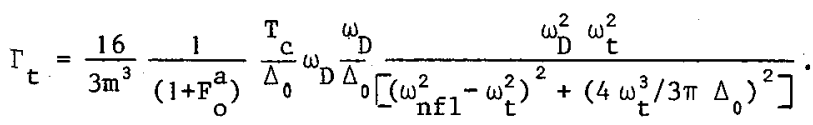
One sees that for decreasing $t \equiv\left(1-T / T_{c}\right)$ the $\Gamma_{t}$ increases as $t^{-1}$ for $\omega_{n f 1} \gg \omega_{t}$, becomes a maximum for $\omega_{n f 1}=\omega_{t}$, and decreases at $t^{2}$ for $\omega_{n f 1} \ll \omega_{t}$. This maximum $\Gamma_{t}$ due to pair-breaking should be compared with the contribution to the line width caused by quasi-particle scattering, that is according to reference $/ 2 /,\left(\omega_{D}^{2} / \omega_{t}^{2}\right)\left(\Gamma_{\|}+\Gamma_{\ell}\right)$ where $\Gamma_{\|}$refers to spin relaxation and $\Gamma_{\ell}$ to orbital effects. According to our estimates the contribution $\Gamma_{t}$ exceeds the latter one. Of course the resonance condition $\omega_{n f 1}=\omega_{t}$ can be satisfied only in high fields and $/$ or $\mathrm{T}$ very close to $\mathrm{T}_{\mathrm{c}}$.

4. EFFECT ON ULTRASOUND ATTENUATION.- From the density correlation function we find for $\vec{q} \| \vec{l}$ in addition to the zero sound mode two normal-flapping and two super-flapping modes whose frequency squares and line widths agree with those given in section 2 apart from the fact that the dipole-flapping terms $\omega_{\ell}^{2}$ are absent. These modes correspond to combined motions of the vectors $\vec{\ell}$ and $\vec{d}$ such that they move in phase. For one type of these modes the vector $\vec{d}$ oscillates in the plane perpendicular to the $\vec{\ell}-\overrightarrow{\mathrm{H}}$ plane, and for the other type the vector $\overrightarrow{\mathrm{d}}$ oscillates in the $\vec{l}-\overrightarrow{\mathrm{H}}$ plane. Therefore the frequency squares of the latter modes, $\omega_{\mathrm{nfl}}^{2}$ and $\omega_{\mathrm{sfl}}^{2}$, contain additional Larmor frequency terms $\omega_{L}^{2}$. There occurs no clapping mode for $\vec{q} \| \vec{l}$ (including $q=0$ ). Although the coupling strength of the flapping modes to the sound wave for $\vec{q} \| \vec{l}$ is only of the order $\mu^{2}$ we find an appreciable effect on the ultrasound attenuation coefficient $\alpha \|$. Considered as a function of temperature $\alpha_{\|}$is peaked at a high temperature, say $T_{1}$, and at a low temperature, say $T_{2}$, where $T_{1}$ and $T_{2}$ are given by the resonance condition $\omega_{\mathrm{nf1}}=\omega$. We find :

$\alpha_{\|} \propto\left(\omega / \mathrm{T}_{\mathrm{c}}\right)\left[1+\left(16 / \pi^{2}\right) 10^{3} \mathrm{~s}^{4} \mu^{2}\left(\mathrm{~T}_{\mathrm{c}} / \omega\right)^{2}\right] ;$

$\left(T_{1} \lesssim T_{c}\right)$.

$\alpha_{\|} \propto\left(\omega / \Delta_{0}\right)^{2}\left[1+\left(2 / \omega^{2}\right) 10^{3} \mathrm{~s}^{4} \mu^{2}\left(\Delta_{0} / \omega\right)^{6}\right] ;$

$\left(T_{2} \ll T_{c}\right)$. 
Here $s=c / v_{F} \simeq 13$ and $\mu \sim 10^{-5}$. The second terms in square brackets yield the relative contribution of the flapping mode in comparison to the ordinary sound attenuation calculated for the collisionless regime. One sees that even near $T_{1}$ the effect of the flapping mode becomes appreciable in spite of the smallness of $\mu^{2}$ provided that $\omega \lesssim 3 \mathrm{MHz}$.

As has been shown by wollfle $/ 4 /$, the coupling strength of the flapping and clapping modes to the sound wave may become much larger than order $\mu^{2}$, i.e., of order $q^{2}$, for orientations of $\vec{q}$ different from $\vec{l}$. Then our theory predicts a relatively narrow absorption peak due to the clapping mode and very broad absorption peaks due to the flapping modes where the latter should split in high magnetic fields.

\section{References}

/1/ Tewordt, L., N. Schopoh1, and .D Vo11hardt, J. Low Temp. Phys. 29 (1977) 119

/2/ Leggett, A.J., and S. Takagi, Annals of Physics 110 (1978) 353

/3/ Combescot, R., Physica 90B (1977) 11

/4/ wö1fle, P., Physica 90B (1977) 96 